Obere Extremität 2012 · 7:5-5

DOI 10.1007/s11678-011-0148-7

Online publiziert: 10. Februar 2012

(c) Springer-Verlag 2012

\section{T. Ambacher}

Schulterchirurgie, ARCUS Kliniken Pforzheim

\section{Schaftfreier Humeruskopfersatz}

Über viele Jahrzehnte war der schaftverankerte Humeruskopfersatz das Standardimplantat zur Versorgung von symptomatischen primären und posttraumatischen Omarthrosen. Insbesondere der zementierte Humeruskopfersatz weist auch bei Langzeitbeobachtungen von größeren Kollektiven über 10-20 Jahre sehr niedrige Komplikationsraten auf. Die Revisionsraten liegen teilweise unter $1 \%$. Durch die Entwicklung modularer Systeme wurde nicht nur die anatomische Wiederherstellung der individuellen Kopfanatomie möglich, sondern zumindest bei einem Teil der Implantate ist auch der Systemwechsel zum inversen Implantat problemlos, wodurch sich der oft mühsame Ausbau des Primärschaftes erübrigt.

Somit schienen zu Beginn der vergangenen Dekade die Probleme des Humeruskopfersatzes gut gelöst. Diskussionen und Entwicklungen fokussierten sich zunächst auf den Glenoidersatz - dieser wird nach wie vor als Schwachpunkt der Schulterendoprothetik angesehen - und auf modulare Implantate zum Wechsel auf das inverse System ohne Schaftausbau. Unterbrochen wurde diese Entwicklung von zwei Herstellern, die 2004 bzw. 2005 schaftfreie Implantate zum Humeruskopfersatz einführten. Ausschlaggebend für diese Entwicklung war einerseits die Überlegung, dass möglicherweise in vielen Fällen eine Schaftverankerung nicht zwingend zur stabilen Implantatfixierung notwendig ist. Andererseits zeigten sich bei posttraumatischen Arthrosen und Kopfnekrosen mit erheblichen Fehlstellungen des Kopfes und der Tuberkula die Grenzen auch der aktuellen Schulterprothesenmodelle, die eine kongruente Abdeckung der Kopfresektionsebene in vielen Fällen nicht erlaubten. Als alterna- tive schaftunabhängige Versorgung wurde häufig der Oberflächenersatz mit all seinen Problemen der schwierigen Implantatplatzierung und Glenoidversorgung verwendet.

Als wesentliche Vorteile des schaftfreien Kopfersatzes wurden knochensparende Versorgung, schaftunabhängige Kopfpositionierung, Reduktion des Risikos für periprothetische Frakturen, geringerer Blutverlust, kürzere Operationszeiten sowie eine einfachere Operationstechnik postuliert. Der Glenoidersatz ist genauso wie bei den Schaftimplantaten möglich. Seit 2004 wurden bislang von vier Herstellern schaftfreie Implantate zum Humeruskopfersatz entwickelt, die aktuell verfügbar sind. Insgesamt wurden weltweit bis Ende 2011 rund 10.000 schaftfreie Humeruskopfimplantate eingesetzt.

Aufgrund der noch jungen Entwicklung liegen aussagekräftige 10-Jahresergebnisse von größeren Fallzahlen noch nicht vor. Diese sind erst im nächsten Jahrzehnt zu erwarten. Die ersten Kongressberichte mit meist kurzen Nachuntersuchungszeiträumen zeigen hinsichtlich des klinischen Ergebnisses mit den Schaftimplantaten vergleichbare Resultate. Ob diese auch längerfristig zuverlässig Bestand haben, werden die noch ausstehenden Nachuntersuchungen zeigen. Systembedingte Probleme der schaftfreien Verankerung sind bislang nicht bekannt. Objektive radiologische Daten hinsichtlich Knochendefekt und -qualität, worin die Grenzen der noch möglichen stabilen Verankerungen der verschiedenen Implantate liegen, gibt es aktuell nicht. Bislang sind die Indikationen abhängig von der subjektiven individuellen Erfahrung und Einschätzung des Operateurs. Die Herausarbeitung objek- tiver Daten zur Frage, bis zu welcher Knochendichte und welchem Defektausmaß noch eine stabile Verankerung schaftfrei möglich ist, wäre wünschenswert und sollte Bestandteil der Nachuntersuchungen sein. Ob sich die schaftfreien Implantate dauerhaft auf breiter Front durchsetzen werden, wird unter anderem von diesen Resultaten abhängen.

Die bisher vorliegenden kurzfristigen Ergebnisse und Erfahrungen mit den Implantaten aller Hersteller sind ermutigend. Die Geschichte der Schulterendoprothetik weist allerdings bereits einige Fehlentwicklungen auf, sodass wir gut beraten sind, die weiteren Resultate und Entwicklung der schaftfreien Humeruskopfimplantate zunächst kritisch zu verfolgen, bevor wir uns ein abschließendes Urteil erlauben.

Ihr

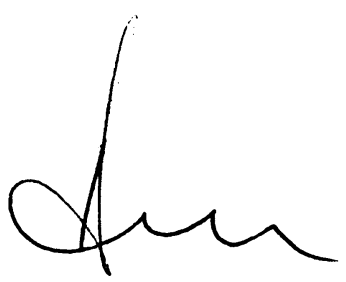

T. Ambacher

\section{Korrespondenzadresse}

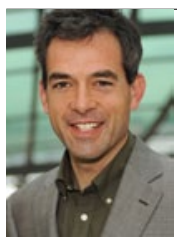

Dr. T. Ambacher

Schulterchirurgie, ARCUS Kliniken Pforzheim Rastatterstrasse 15-17, 75179 Pforzheim ambacher@sportklinik.de 